\title{
HUMEDAD Y SU RELACIÓN CON LA ESPECTROSCOPÍA DIELÉCTRICA EN AGREGADOS DE CONCRETO
}

\section{Moisture in Concrete Aggregates And ITS RELATION TO THE DIELECTRIC CONSTANT}

\author{
Franco Abanto ${ }^{1, *}$, Pedro Rotta ${ }^{1}$, Luis LaMadrid ${ }^{1}$, Juan Soto $^{1}$, Gerson La Rosa ${ }^{1}$, \\ José Manrique $^{1}$, Gaby Ruiz ${ }^{1}$, William Ipanaque ${ }^{1}$
}

\section{Resumen}

Medir el contenido de humedad $(\mathrm{CH})$ de los agregados de concreto (ADC) en la fabricación de concreto premezclado es uno de los retos en la industria de la construcción porque afecta a las propiedades finales del concreto. Actualmente los métodos que se utilizan para medir el $\mathrm{CH}$ en ADC son invasivos y destructivos. Este artículo presenta una técnica moderna basada en espectroscopía dieléctrica (ED), un método que al propagar microondas en el material correlaciona su constante dieléctrica (CD) y su CH. En esta investigación se ha utilizado este método en ADC. Tres diferentes canteras peruanas de ADC (Moyobamba, Sol-Sol y Cerro Mocho) han sido utilizadas. Los resultados demuestran que el sensor a una frecuencia de $1.5 \mathrm{GHz}$ es capaz de detectar el $\mathrm{CH}$ en ADC con una regresión lineal de $\mathrm{R}^{2}=95 \%$. En conclusión, se puede utilizar la ED como un método de sensado no invasivo y en línea de $\mathrm{CH}$ en ADC para ser utilizado en la industria de la construcción.

Palabras clave: contenido de humedad, microondas, espectroscopía dieléctrica, constante dieléctrica, agregados de concreto

\section{Abstract}

Measurement of moisture content $(\mathrm{CH})$ of concrete aggregates $(\mathrm{AOC})$ in the manufactury of ready mixed concrete is one of the currently challenges in the building industry since affect to the final properties of concret. At present, the methods for measurement of $\mathrm{CH}$ in AOC are invasive and destructive. This paper presents a novel sensing technique using dielectric espectroscopy (ED), a method that using the propagation of microwaves on the material allows the correlation of its dielectric constant (CD) and its $\mathrm{CH}$. In this research is used this method in AOC. Three diferents peruvian quarries (Moyobamba, Sol sol y Cerro Mocho) have been used. The results shows that the sensor at the frequency of $1.5 \mathrm{GHz}$ is capable of detecting the $\mathrm{CH}$ in $\mathrm{AOC}$ with linear regression of $\mathrm{R}^{2}=95 \%$. In conclusion, is available using the ED as a online and no invasive sensing method of $\mathrm{CH}$ in AOC for using in the building industry.

Keywords: moisture contentent, microwaves, dielectric spectroscopy, dielectric contant, concrete aggregates.

\footnotetext{
${ }_{1, *}$ Laboratorio de Sistemas Automáticos de Control, Universidad de Piura, Perú.

Autor para correspondencia fabanto977@hotmail.es.

(D) https://orcid.org/0000-0002-9388-2692 (D) https://orcid.org/0000-0002-6439-2870

(D) https://orcid.org/0000-0002-4293-8086 (D) https://orcid.org/0000-0001-9157-3098

(D) https://orcid.org/0000-0001-6829-2706 (D) https://orcid.org/0000-0002-0331-2734

(D) https://orcid.org/0000-0003-3835-9708 (iD https://orcid.org/0000-0003-4039-4422
}

Recibido: 14-10-2019, aprobado tras revisión: 13-04-2020

Forma sugerida de citación: Abanto, F.; Rotta, P.; LaMadrid, L.; Soto, J.; La Rosa, G.; Manrique, J.; Ruiz, G. y Ipanaque, W. (2020). «Humedad y su relación con la espectroscopía dieléctrica en agregados de concreto». INGENIUS. N. ${ }^{\circ} 24$, (julio-diciembre). pp. 17-27. DOI: https://doi.org/10.17163/ings.n24.2020.02. 


\section{Introducción}

El contenido de humedad $(\mathrm{CH})$ de un material es un parámetro que muchos sectores industriales buscan controlar en sus procesos, pues repercute en las características finales del producto. En la industria de la construcción, el CH del concreto resulta importante porque define las propiedades mecánicas y la vida útil de un proyecto civil [1]. Se han realizado estudios para analizar la durabilidad y resistencia en estructuras de concreto midiendo el $\mathrm{CH}$ [2] y también en probetas de concreto [3], no se han realizado investigaciones de sistemas que midan el $\mathrm{CH}$ de $\mathrm{ADC}$ en línea con el proceso de mezclado en planta. Autores en [4-6] muestran diferentes técnicas para lograr la medición del $\mathrm{CH}$ de materiales. En el presente artículo se utiliza una metodología basada en espectroscopía dieléctrica (ED) que ha sido probada en suelo [7], lana [8], papel [9], tela [10], harina [11], madera [12-14].

Existen varios métodos de medición de $\mathrm{CH}$ de materiales que se clasifican en directos e indirectos. En los métodos directos se obtiene el $\mathrm{CH}$ sin correlacionar con otras variables. Estos son los métodos termogavimétrico y químico. El método termogavimétrico no es selectivo $[15,16]$, el rango eficaz de medición varía de $0.5 \%$ a $99.9 \%$ para el $\mathrm{CH}$, y su exactitud es de $0.5 \%$ de la masa total. En contraste, el método químico [17-19] es selectivo, tiene una precisión de $0.0001 \%$ y un rango de medición de 0.00001 a $99.9 \%$ de $\mathrm{CH}$.

Los métodos indirectos requieren una calibración previa para obtener el $\mathrm{CH}$ usando métodos directos. Los indirectos se clasifican en pasivos y activos. Los primeros utilizan elementos como resistencias o capacitancias variables para determinar la $\mathrm{CH}$, y por su naturaleza es un control invasivo. Los activos son aquellos que emiten ondas electromagnéticas para determinar las características del medio, garantizando de esta manera un control en línea, y la integridad de la muestra al no ser invasivo ni destructivo.

Los resultados de las diversas investigaciones en [20-23] dentro del campo de los métodos activos, demuestra, una relación existente entre el $\mathrm{CH}$ y la permitividad relativa $\left(\varepsilon^{\prime}\right)$ o constante dieléctrica $(\mathrm{CD})$ [24] de un material.

Los métodos indirectos usan técnicas como la ED que busca medir la CD del material y también se utiliza para otros fines como caracterizar materiales. Otra técnica indirecta es el uso de imágenes hiperespectrales que ha tenido buen éxito en bioingeniería [25] y en agroindustria [26-29].

Autores muestran aplicaciones con ED orientadas a la agricultura con el fin de estimar la calidad de sus productos [30-41], así tenemos aplicaciones en semillas, trigo, granos, nueces, frutos de palma aceitera, plátanos.

Este artículo describe conceptos teóricos de la ED y su relación con el CH [6]. Se presenta una aplicación de la ED para buscar la correlación entre el CH y la $\mathrm{CD}$ en $\mathrm{ADC}$ con diferentes canteras y verificar la posibilidad de este nuevo método en esta industria.

\subsection{Descripción de las ondas electromagnéticas}

Se denomina campos electromagnéticos al conjunto de campos de fuerzas eléctricas y magnéticas producidas por cargas y corrientes eléctricas en movimiento a través del vacío o algún tipo de materia. Cuando un campo electromagnético se propaga en el espacio se denomina propagación de ondas electromagnéticas.

La propagación de ondas electromagnéticas encuentra su fundamento en la solución de las ecuaciones de Maxwell.

$$
\begin{gathered}
\left.\nabla \times \bar{E}=\bar{M}-\frac{\partial \bar{B}}{\partial t} \text { (ley de Faraday }\right) \\
\nabla \times \bar{H}=\bar{J}-\frac{\partial \bar{D}}{\partial t}(\text { ley de Ampere }) \\
V \cdot \bar{D}=\rho(\text { Ley de Gauss }) \\
V \cdot \bar{H}=0(\text { Ley de Gauss })
\end{gathered}
$$

Donde:

$E$ es el campo eléctrico $[\mathrm{V} / \mathrm{m}]$

$H$ es el campo magnético $[\mathrm{A} / \mathrm{m}]$

$M$ es la densidad de corriente magnética $\left[\mathrm{V} / \mathrm{m}^{2}\right]$

$J$ es la densidad de corriente eléctrica $\left[\mathrm{A} / \mathrm{m}^{2}\right]$

$B$ es la densidad de flujo magnético $\left[\mathrm{Wb} / \mathrm{m}^{2}\right]$

$D$ es la densidad de flujo eléctrico $\left[\mathrm{Coul} / \mathrm{m}^{2}\right]$

$\rho$ es la densidad de carga $\left[\mathrm{Coul} / \mathrm{m}^{3}\right]$

Para resolver las ecuaciones de Maxwell se supone propagación en el espacio libre y, además, un campo senoidal y armónico dependiente del tiempo, que se propague en el eje z y polarizado en el eje $\mathrm{x}$.

Al utilizar estas suposiciones y combinar las ecuaciones dadas se genera una ecuación de segundo orden que se conoce como ecuación vectorial homogénea de Helmholtz para E.

$$
\nabla^{2} E+k^{2} E=0
$$

Donde $\mathrm{k}$ es el número de onda que para un medio sin pérdida y se expresa como:

$$
k=\omega \sqrt{\varepsilon_{0} \mu_{0}}=\frac{\omega}{c_{0}}=\frac{2 \pi}{\lambda}\left(\frac{\mathrm{rad}}{\min }\right)
$$

Donde:

$\omega$ es la frecuencia angular de propagación

$\varepsilon_{0}$ es la permitividad del vacío

$\mu_{0}$ es la permeabilidad del vacío 
Resolviendo:

$$
\bar{E}(z, t)=\overline{a_{x}} \varepsilon_{0} \cos (\omega t-k z)
$$

que toma el valor fasorial:

$$
\bar{\varepsilon}(z)=\overline{a_{x}} \varepsilon_{0} e^{-j k z}
$$

Para expresar de forma senoidal, el vector fasorial se puede utilizar la siguiente expresión:

$$
\bar{E}(z, t)=\operatorname{Re}\left\{\bar{\varepsilon}(z) e^{j \omega t}\right\}
$$

En la Figura 1 se puede observar la propagación del campo eléctrico en el espacio libre, en donde se han tomado las hipótesis dadas.

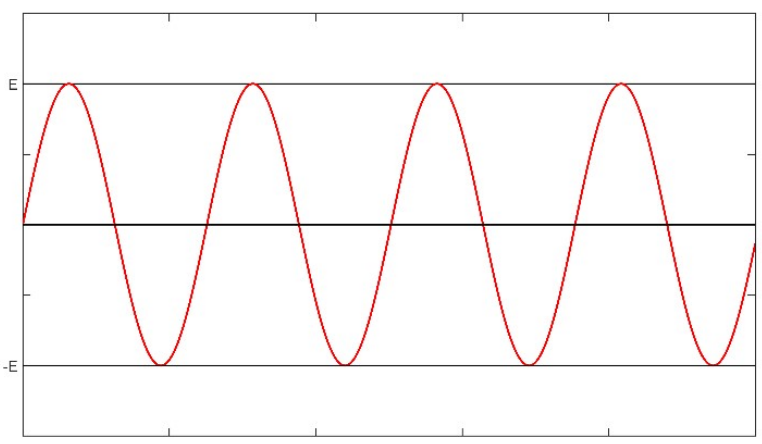

Figura 1. Representación de una onda electromagnética que viaja en el espacio libre [11]

\subsection{Propagación de las ondas en un medio con pérdidas}

Las hipótesis definidas en el apartado anterior consideran la propagación de ondas electromagnéticas en el vacío. Se amplían a materiales con pérdidas, es decir, materiales convencionales.

Los materiales se clasifican según su comportamiento frente a campos como buenos conductores cuando permiten el paso de campos eléctricos por ellos o como dieléctricos cuando almacenan energía eléctrica en su interior y forman enlaces moleculares polares que se conocen como dipolos eléctricos. Normalmente, un material tiene una parte conductora y otra dieléctrica. $\mathrm{Su}$ comportamiento se determina mediante la permitividad compleja del material, que se define como:

$$
\varepsilon_{c}=\varepsilon^{\prime}-j \varepsilon^{\prime \prime}
$$

Donde $\varepsilon^{\prime}$ es la constante dieléctrica del material y mide la cantidad de momentos dipolares formados y representa la energía contenida en el material y $\varepsilon^{\prime \prime}$ es la constante de pérdidas que representa la energía que no es almacenada en el material, sino que de alguna manera se propaga o refleja y se representa como:

$$
\varepsilon^{\prime \prime}=\varepsilon_{r}^{\prime \prime}+\frac{\sigma}{\omega}
$$

Donde $\sigma$ es la conductividad del material tal que:

$$
\bar{J}=\sigma \bar{E}
$$

Se obtiene una conductividad equivalente que representa todas las pérdidas en el medio.

$$
\sigma_{e q}=\omega \varepsilon^{\prime \prime}
$$

La «tangente de pérdidas» es una medida de la pérdida de potencia en el medio y se define.

$$
\tan \delta=\frac{\varepsilon^{\prime \prime}}{\varepsilon^{\prime}}=\frac{\omega \varepsilon^{\prime \prime}+\sigma}{\omega \varepsilon^{\prime}}
$$

Por lo tanto, la solución de las ecuaciones de Maxwell a través de la ecuación vectorial homogénea de Helmholtz para $E$ queda de la forma:

$$
\begin{gathered}
\nabla^{2} \vec{E}+k_{c}^{2} \vec{E}=0 \\
k_{c}=\omega \sqrt{\varepsilon_{c} \mu}\left(m^{-1}\right)
\end{gathered}
$$

Donde $k_{c}$ es el número de onda complejo, es decir, que toma el valor complejo de la permitividad del medio que se comporta de modo fasorial al tomar un campo eléctrico senoidal. Además, la permitividad del vacío queda expresada como un valor real, ya que no tendrá pérdidas:

$$
\varepsilon_{0}=8.854 \times 10^{-12} \frac{F}{m}
$$

Por lo tanto, esta forma de comportamiento de los materiales hace que las ondas electromagnéticas se atenúen al momento de incidir en ellas, parte de la energía será almacenada en los enlaces polares y parte se irá en pérdidas de energía. Esto hace que se defina el concepto de constante de propagación como:

$$
y=\alpha+j \beta=j \omega \sqrt{\mu \varepsilon^{\prime}}\left(1+\frac{\sigma}{j \omega \varepsilon}\right)^{\frac{1}{2}}
$$

Usando la definición de pérdida tangencial.

$$
y=\alpha+j \beta=j \omega \sqrt{\mu \varepsilon^{\prime}}\left(1-j \frac{\varepsilon^{\prime \prime}}{\varepsilon^{\prime}}\right)^{\frac{1}{2}}
$$

Donde $\alpha$ es la constante de atenuación y $\beta$ es la constante de fase.

Entonces la solución primaria que se da en el vacío queda como:

$$
\bar{E}(z, t)=\overline{a_{x}} \varepsilon_{0} \cos (\omega t-\beta z)
$$

que toma el valor fasorial:

$$
\bar{\varepsilon}(z)=\overline{a_{x}} \varepsilon_{0} e^{-j \beta z}
$$

Donde:

$$
\bar{E}(z, t)=\operatorname{Re}\left\{\bar{\varepsilon}(z) e^{j \omega t}\right\}
$$


En la Figura 2 se muestra la representación de esta atenuación de un campo eléctrico que incide en un material con pérdidas.

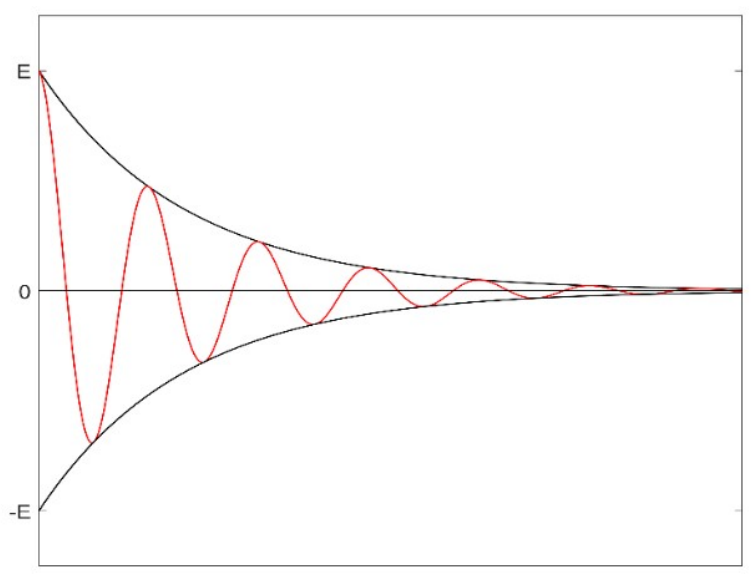

Figura 2. Representación de un campo eléctrico que viaja en un medio con pérdidas [11]
La energía perdida al momento de la propagación sobre el material, recibe ese nombre porque no se almacena en el material formando enlaces polares, parte de esta energía se refleja del material y parte de ella atraviesa el material, de acuerdo con el valor de conductividad de este. Esto se aprecia en la Figura 3. Se observa un campo eléctrico incidente de verde que al chocar en el medio (de rayas azules) se refleja parte del campo (de rojo) y parte se propaga por el campo (de naranja).

Todo esto queda cuantificado en la permitividad compleja.

En todo este análisis se asume que el material es isotrópico, es decir, que los momentos dipolares o que los enlaces polares se dan en la dirección del campo eléctrico, en materiales anisotrópicos esto no ocurre, pero este análisis no se toma en cuenta para esta investigación ya que hemos considerado a los ADC isotrópicos.

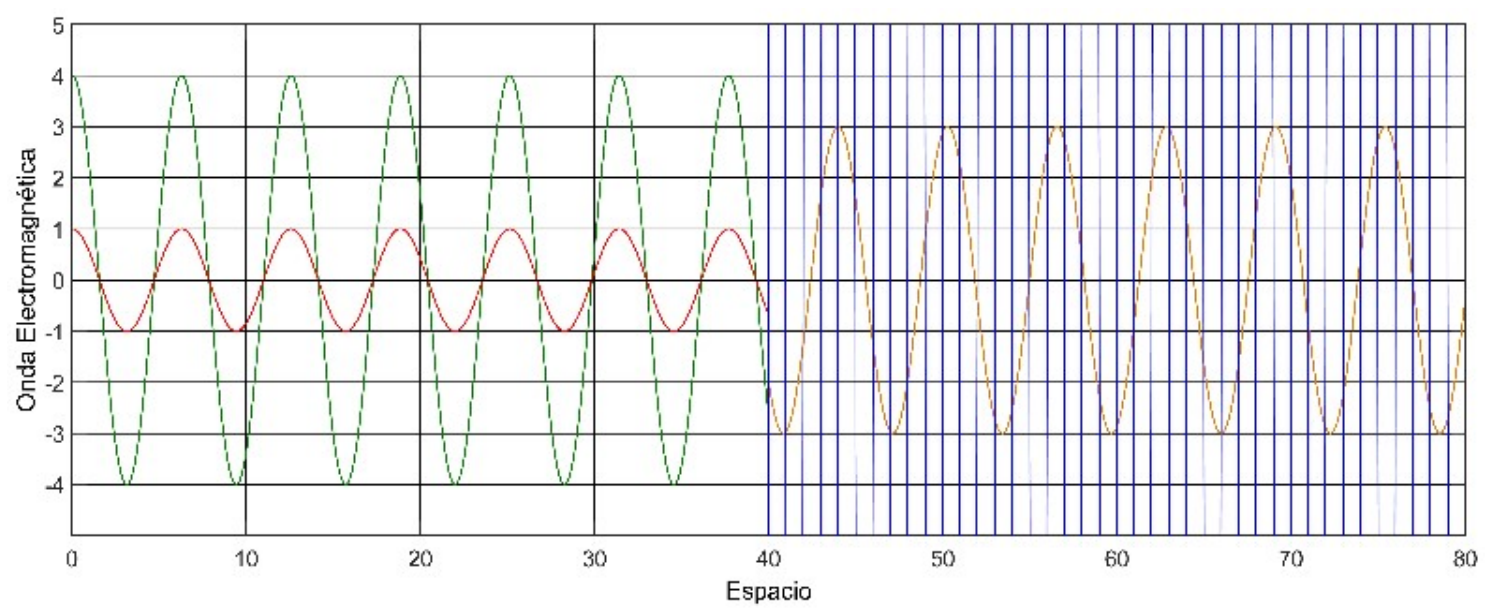

Figura 3. Comportamiento de la propagación de una onda electromagnética frente a un cambio de medio [11]

La parte del campo reflejado se puede relacionar respecto al campo incidente mediante el coeficiente de reflexión $\Gamma$ que relaciona la onda reflejada y la onda incidente del campo.

$$
\Gamma=\frac{E_{r}}{E_{i}}
$$

Reemplazando la ecuación de la onda para expresarlo en términos de campo eléctrico, sería:

$$
E_{r}(z)=\Gamma E_{0} e^{y_{1} z} \alpha_{x}
$$

La ecuación del campo magnético de la onda propagada y reflejada se dirige en la dirección ortogonal al campo eléctrico:

$$
H_{r}(z)=\Gamma \frac{E_{0}}{n_{1}} e^{y_{1} z}\left(-a_{y}\right)
$$

Es posible medir el campo eléctrico incidente en un material, el campo propagado y el campo reflejado de acuerdo con las ecuaciones dadas en la teoría. Con todo esto podemos asumir que es posible deducir los valores de $\mathrm{CD}$ que tomará el material al analizar la relaciones entre estas cantidades.

\subsection{Propiedades dieléctricas de la molécula de agua}

El agua es un dieléctrico, es decir, como hemos visto contiene en su estructura moléculas polares que forman momentos dipolares al estar en contacto con un campo eléctrico y por ende a mayor cantidad de agua, mayor será la CD medida.

Un material seco tendrá un comportamiento establecido según su estructura molecular y será nor- 
malmente homogéneo cuando esta estructura se mantenga inalterable al sufrir aumento de temperatura o mezclarse con agua. Los ADC, por su forma y propiedades, presentan una estructura homogénea. Por ende, su constante dieléctrica permanecerá inalterable cuando se humedezca. Sin embargo, a mayor humedad se incrementará el momento dipolar de la mezcla por el agua presente, lo que ocasionará que la $\mathrm{CD}$ de la mezcla cambie debido al aumento de agua. Por lo tanto, la $\mathrm{CD}$ de la mezcla se verá relacionada con el $\mathrm{CH}$ de los ADC y si podemos medir el $\mathrm{CH}$ y la $\mathrm{CD}$ de la mezcla podemos determinar una correlación entre estas para una futura predicción y utilizarla como sistema de sensado.

\section{Metodología}

Se ha visto en el apartado anterior que es posible correlacionar el valor del $\mathrm{CH}$ de los $\mathrm{ADC}$ con la $\mathrm{CD}$ de la mezcla ya que la cantidad de momentos dipolares aumentará de acuerdo con su CH. Además, se ha visto teóricamente que es posible determinar la CD de la mezcla utilizando las ecuaciones de Maxwell y su solución para medios con pérdidas. En este apartado se brinda la metodología experimental que se ha seguido para realizar dicha correlación.

Es preciso recalcar que el campo emitido es el campo que choca con el material, y el campo propagado es el que atraviesa el material.

\subsection{Materiales}

La ED usa frecuencias en el rango de las microondas para la propagación de los campos electromagnéticos, por lo tanto, se utilizan dos antenas de apertura para emitir y recibir los campos incidente y propagado respectivamente. Ver Figura 4.

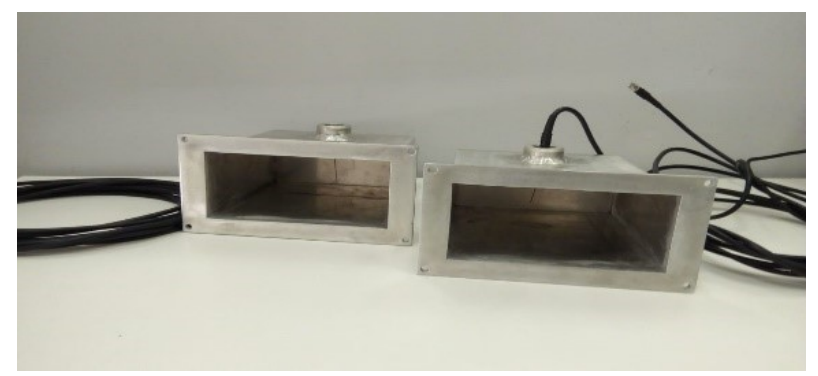

Figura 4. Antenas de apertura utilizadas en pruebas de medición de $\mathrm{CH}$

También se ha utilizado un sistema de análisis de señales vectoriales para emitir el campo electromagnético como se ve en la Figura 5. Se analiza la variación de la fase y amplitud de la señal para posteriormente determinar la CD.

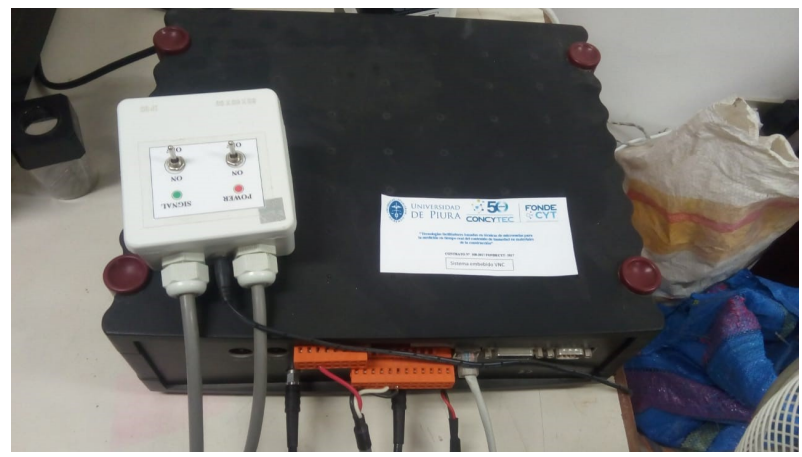

Figura 5. Equipo analizador de señales vectoriales Wavetester

El analizador de señales vectoriales utiliza un software para detección de datos.

También se ha construido una plataforma de sensado para realizar la experimentación, sobre la que se ha colocado el ADC para medir su CD y su CH. Otros materiales utilizados han sido: balanzas, recipientes de medición, horno de secado, etc.

La muestra se deposita entre la antena receptora y la transmisora, donde se mide el efecto de la onda sobre los ADC al propagarse en el espacio libre entre las dos antenas.

\subsection{Experimentación}

Se han utilizado tres canteras peruanas de ADC para realizar la calibración del sistema: Cerro Mocho, Moyobamba y Sol-Sol. Para obtener la correlación CH vs. $\mathrm{CD}$ se ha proseguido de la siguiente manera:

Se ha definido una masa inicial $\left(m_{0}\right)$ como la masa total de ADC proporcionado por la cantera. Luego se ha realizado un secado termogavimétrico en horno para obtener el valor de masa seca $\left(m_{s}\right)$, esto quiere decir, sin CH. Este valor de $m_{s}$ se ha dividido en 4, cada una de estas muestras se ha denominado masa seca de muestreo y se han numerado del 1 al $4\left(m_{s m x}\right)$, el $\mathrm{x}$ en el subíndice corresponde a la numeración de la submuestra. Se procede a seleccionar la $m_{s m 1}$ y se coloca sobre la plataforma de sensado, se emite el campo electromagnético sobre el material y con ayuda del analizador de señales se mide el valor de la CD de la $m_{s m 1}$. Este valor de CD corresponde al valor de $0 \%$ de $\mathrm{CH}$. A este valor de $m_{s m 1}$ se le agrega masa de agua $\left(m_{H_{2} \mathrm{O}}\right)$ correspondiente a $0.5 \% m_{s m 1}$ y se procede a realizar el mismo procedimiento de medición de su CD. Luego se vuelve a añadir $0.5 \% m_{s m 1}$ y se mide su CD que corresponde al $1 \%$ de su CH. Este procedimiento se repite hasta que se llega al $10 \%$ de $\mathrm{CH}$.

$$
m_{h 2 O}=0.005 \times m_{s m 1}
$$

Se debe aclarar que se cumplen las siguientes relaciones en la experimentación: 


$$
\begin{gathered}
m_{s m 1}=m_{s m 2}=m_{s m 3}=m_{s m 4} \\
m_{s m 1}+m_{s m 2}+m_{s m 3}+m_{s m 4}=m_{s}
\end{gathered}
$$

La distancia entre antenas fue de $23 \mathrm{~cm}$, el espesor de la muestra se estableció en $40 \mathrm{~mm}$ y la frecuencia de emisión del campo electromagnético fue de $1.5 \mathrm{GHz}$.

Se utilizaron $m_{s m 1}$ y $m_{s m 2}$ para realizar las curvas de correlación y $m_{s m 3}$ y $m_{s m 4}$ para validar los resultados. Cabe resaltar que en todo momento se mide la $\mathrm{CD}$ de la mezcla de ADC húmedo.

\section{Resultados}

Con los valores de $\mathrm{CD}$ vs. $\mathrm{CH}$ obtenidos para cada cantera se ajusta la curva de calibración mediante modelos de regresión lineal. En este ajuste el $\mathrm{CH}$ se establece como variable dependiente y la CD como variable independiente con diferentes efectos: lineal, cuadrática, cúbica y de cuarto grado.

Para la selección del modelo de regresión lineal se aplicó el método Stepwise Forward para encontrar cuál de los efectos de la CD se ajusta mejor con el CH. Este método clásico para la selección de variables inicia con un modelo vacío y en cada iteración evalúa incorporar algunos de los efectos definidos: lineal, cuadrático, cúbico y de cuarto grado de la constante dieléctrica. Se decide incorporar algunos de los efectos mencionados si cumple el nivel de significancia definido: Valor P menor a 0,05. El método Stepwise Forward finaliza cuando no se puede incorporar más efectos por no cumplir el nivel de significancia.

Para evaluar el nivel de significancia de los efectos se realiza una prueba de hipótesis con T-Student. En esta prueba se verifica si el coeficiente estimado del efecto es igual o diferente de cero.

$$
\begin{aligned}
& H_{0}: b_{i}=0 \\
& H_{1}: b_{i} \neq 0
\end{aligned}
$$

Si se rechaza la hipótesis nula $\left(b_{i} \neq 0\right)$ el efecto es significativo.

En el contraste de hipótesis se calcula la relación entre el coeficiente estimado del efecto $\left(b_{i}\right)$ y su desviación estándar $\left(S_{b_{i}}\right)$, y se compara con el $t$ crítico para un nivel de confiabilidad de $95 \%(\alpha=0,05)$.

$$
\frac{b_{i}}{S_{b i}}>j_{N-1}^{\alpha / 2}
$$

Si se cumple la relación se rechaza la hipótesis nula. En esta condición se cumple que el «valor $\mathrm{P}$ » es menor a 0.05 .

En el ajuste del modelo de regresión también se vio necesario aplicar el procedimiento iterativo de
Cochrane-Orcutt para corregir la autocorrelación presente en los datos. Esta autocorrelación es producto de agregar secuencialmente la variación de humedad y con esta corrección se mejora la estimación de los parámetros.

Los resultados de las pruebas se presentan a continuación.

\subsection{Cantera Cerro Mocho}

El modelo se seleccionó mediante Stepwise Forward, donde se obtiene:

$$
(C H \%)=-7.372+3.206 \times C D
$$

En este modelo, el efecto lineal del coeficiente dieléctrico con respecto al valor de humedad esperado resulta significativo. En la tabla siguiente se muestran los resultados del contraste de hipótesis donde el «valor $\mathrm{P}$ » del efecto lineal es menor a 0.05 . Se tiene un modelo de regresión lineal de $\mathrm{R}^{2}=95.8057 \%$ y un error estándar de 0.382134 .

Tabla 1. Significancia del efecto de las variables

\begin{tabular}{ccccc}
\hline Parámetro & $\begin{array}{c}\text { Valor } \\
\text { estimado }\end{array}$ & $\begin{array}{c}\text { Error } \\
\text { estándar }\end{array}$ & T-Student & Valor P \\
\hline Constante & 7.37238 & 0.378829 & 19.461 & 0.000 \\
Cte_Dieléctrica & 3.2059 & 0.0740563 & 43.29 & 0.000 \\
\hline
\end{tabular}

La Figura 6 muestra la relación entre la constante dieléctrica y la humedad; la Figura 7, la relación del valor predicho y el real de la humedad.

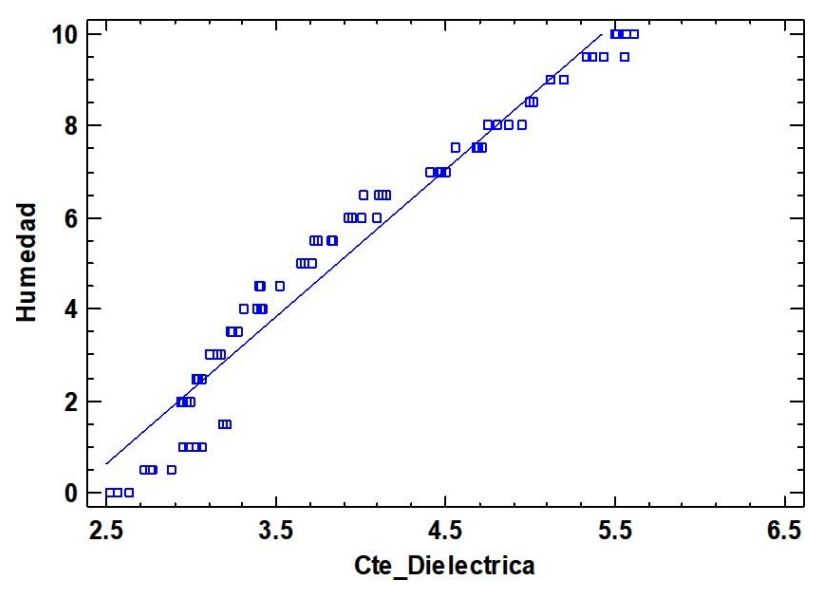

Figura 6. Gráfico del modelo ajustado 


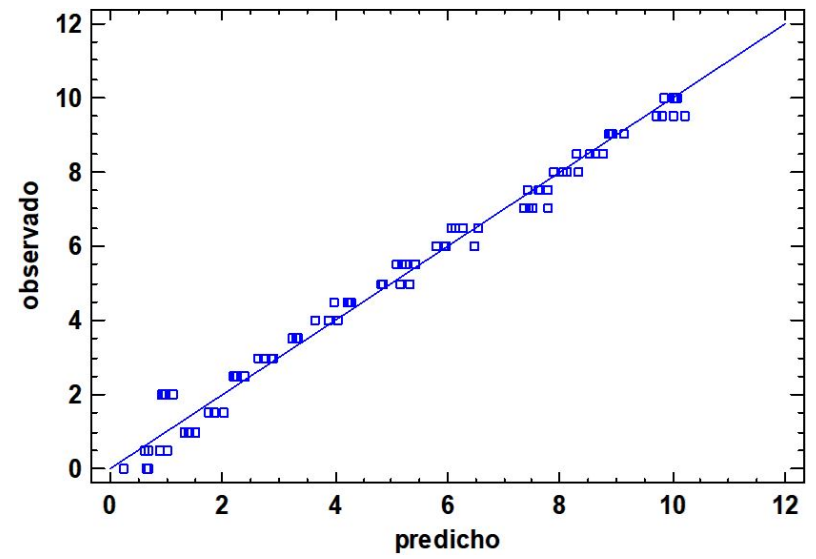

Figura 7. Relación gráfica entre el valor de humedad observado y el predicho

\subsection{Cantera Moyobamba}

El modelo se seleccionó mediante Stepwise Forward.

Donde el efecto lineal, cuadrático y cúbico del coeficiente dieléctrico con respecto al valor de humedad esperado son significativos.

$(C H \%)=38.55+18.15 \times C D-2.52 \times C D^{2}+0.13 \times C D^{3}$

En la Tabla 2 se muestran los resultados del contraste de hipótesis donde el «valor $\mathrm{P} »$ de los efectos es menor a 0.05 .

Tabla 2. Significancia del efecto de las variables

\begin{tabular}{ccccc}
\hline Parámetro & $\begin{array}{c}\text { Valor } \\
\text { estimado }\end{array}$ & $\begin{array}{c}\text { Error } \\
\text { estándar }\end{array}$ & T-Student & Valor P \\
\hline Constante & 38.55 & 1.93216 & 19.9518 & 0.000 \\
Cte_Dieléctrica & 18.1473 & 1.045 & 17.3657 & 0.000 \\
Cte_Dieléctrica2 & 2.52011 & 0.18239 & 13.8172 & 0.000 \\
Cte_Dieléctrica3 & 0.1253 & 0.0102973 & 12.1681 & 0.000 \\
\hline
\end{tabular}

Se tiene un modelo de regresión lineal de $\mathrm{R}^{2}=$ $99.5097 \%$ y un error estándar de 0.201714 .

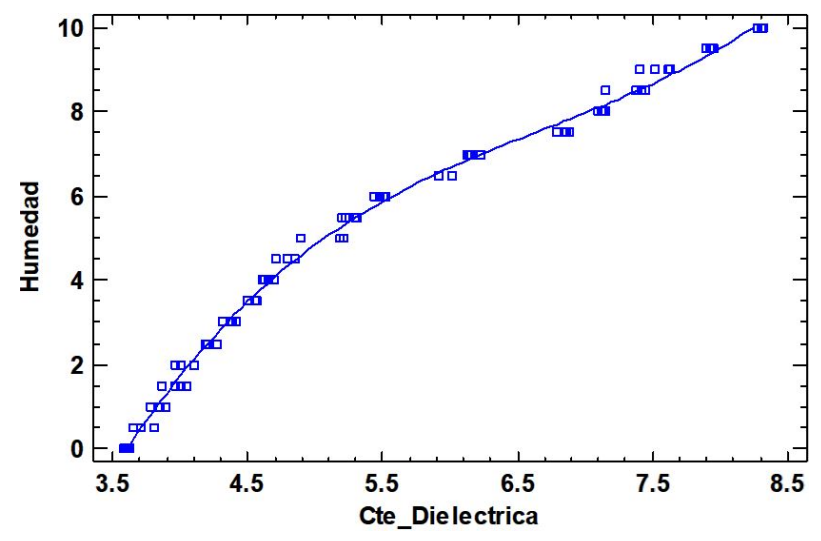

Figura 8. Gráfico del modelo ajustado

La Figura 8 muestra la relación entre la constante dieléctrica y la humedad; la Figura 9 indica la relación del valor predicho y el real de la humedad.

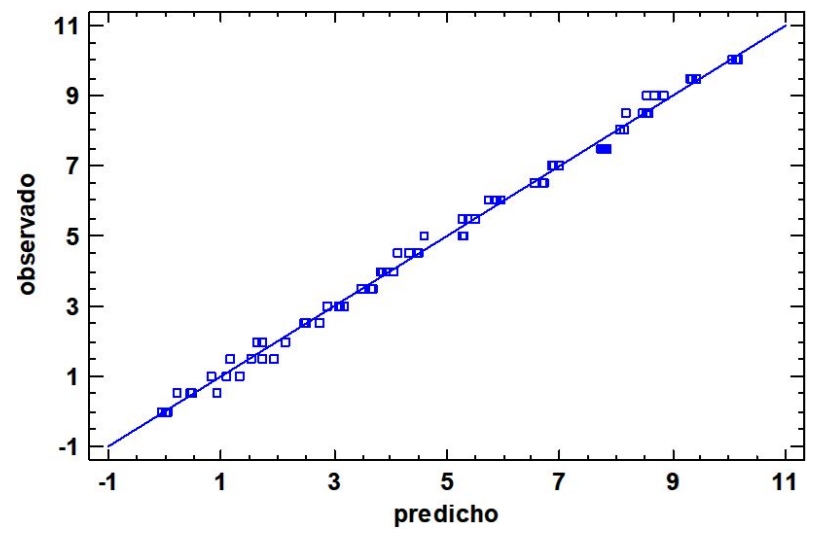

Figura 9. Relación gráfica entre el valor de humedad observado y el predicho

\subsection{Cantera Sol-Sol}

El modelo se seleccionó mediante Stepwise Forward.

$(C H \%)=-15.5262+7.26581 \times C D-0.506717 \times C D^{2}$

Donde el efecto lineal y cuadrático del coeficiente dieléctrico con respecto al valor de humedad resultaron significativos. En la Tabla 3 observamos que el «valor $\mathrm{P} \gg$ de los efectos es menor a 0.05 .

Tabla 3. Significancia del efecto de las variables

\begin{tabular}{ccccc}
\hline Parámetro & $\begin{array}{c}\text { Valor } \\
\text { estimado }\end{array}$ & $\begin{array}{c}\text { Error } \\
\text { estándar }\end{array}$ & T-Student & Valor P \\
\hline Constante & -15.5262 & 0.875589 & 17.7323 & 0.000 \\
Cte_Dieléctrica & 7.26581 & 0.41318 & 17.5869 & 0.000 \\
Cte_Dieléctrica2 & 0.506717 & 0.0477948 & 10.6011 & 0.000 \\
\hline
\end{tabular}

Se tiene un modelo de regresión lineal de $\mathrm{R}^{2}=97.1325 \%$ y un error estándar de 0.297068.

La Figura 10 muestra la relación entre la constante dieléctrica y la humedad, mientras que en la Figura 11 se observa la relación del valor predicho y el real de la humedad.

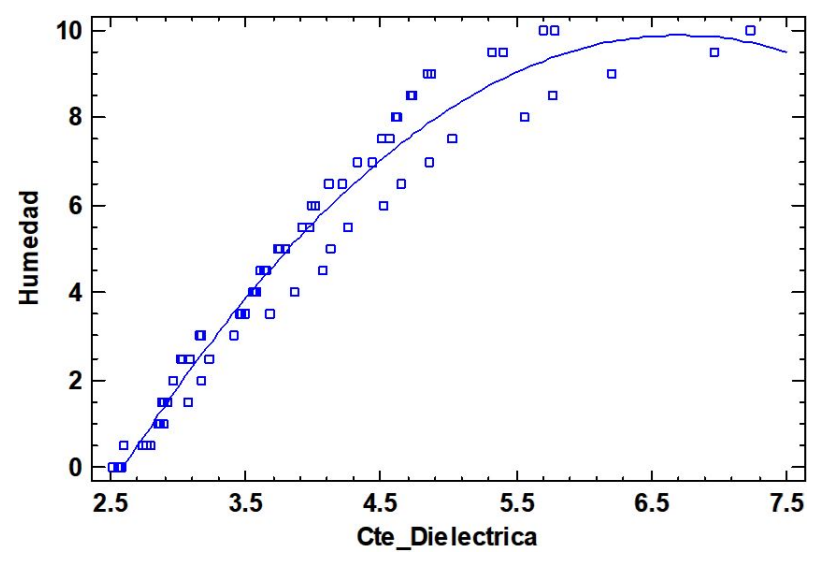

Figura 10. Gráfico del modelo ajustado 


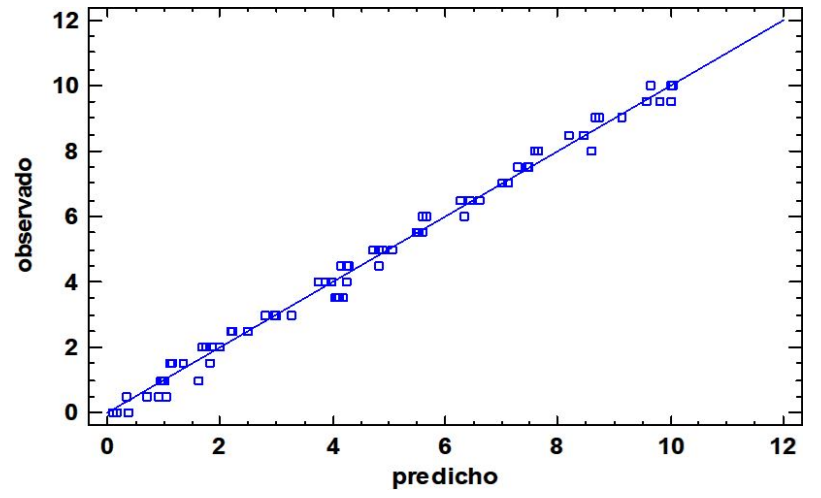

Figura 11. Relación gráfica entre el valor de humedad observado y el predicho

\section{Discusión de resultados}

Dados los resultados en el apartado anterior, es interesante ver que en la frecuencia de $1.5 \mathrm{GHz}$, las correlaciones de regresión lineal mantienen un $\mathrm{R}^{2}>95 \%$ como se ve en la Tabla 4 .

Tabla 4. Comparación de resultados

\begin{tabular}{ccc}
\hline Cantera & $\mathbf{R}^{2}$ & Error estándar \\
\hline Cerro Mocho & 95.8057 & 0.382134 \\
Moyobamba & 99.5097 & 0.201714 \\
Sol-Sol & 97.1325 & 0.297068 \\
\hline
\end{tabular}

También se puede ver que existe una relación directa entre el $\mathrm{CH}$ y la $\mathrm{CD}$, es decir, a mayor $\mathrm{CH}$ la $\mathrm{CD}$ aumenta en valor.

Comparando las ecuaciones de predicción del $\mathrm{CH}$ podemos observar que dependiendo de la procedencia del ADC se define su curva de calibración y esta puede variar entre lineal, cuadrática o cúbica, por lo que para fines prácticos primero se debería calibrar el ADC según una cantera específica antes de realizar la medición y esta curva no serviría para otra cantera, ya que los valores de $\mathrm{CD}$ difieren entre canteras. Esto era de esperarse ya que la CD depende de las propiedades moleculares y de la capacidad de almacenamiento de energía, lo que significa que a nivel molecular cada ADC tiene diferente estructura.

\section{Conclusiones}

La medición del $\mathrm{CH}$ con dispositivos que utilizan microondas tiene ventajas sobre los métodos invasivos porque no dañan el material. La medición de la CD con esta metodología analiza internamente el comportamiento del material para definir su CD, ya que estudia los momentos dipolares formados al inducir campos electromagnéticos en el material. Puede ser usado en presencia de vapores o ambientes sucios, mientras no cambien molecularmente al ADC ya que estos no interfieren con las señales de microondas. La ED, por lo tanto, permite la medición de una amplia gama de materiales, ya sean sólidos, gases o líquidos.

La medición se realiza sin contacto con el material. El método es no invasivo ni destructivo. La medición se realiza en tiempo real y en línea con el proceso.

Es interesante observar la relación encontrada por diferentes autores. En [17] el autor define una relación lineal o polinómica. Muestra los parámetros que influyen en el cálculo de la constante dieléctrica y la relación entre el contenido de humedad y la temperatura.

Se ha comprobado que con la metodología basada en $\mathrm{ED}$ a $1.5 \mathrm{GHz}$ se obtienen valores de correlación lineal de alta precisión $\left(\mathrm{R}^{2}>95 \%\right)$ para cada una de las canteras. El sistema ha sido validado en un transportador horizontal con agregado fino y antenas dispuestas de forma vertical.

Los resultados encontrados muestran la relación entre el $\mathrm{CH}$ y la $\mathrm{CD}$ en $\mathrm{ADC}$, se ha podido apreciar que existe una variación en la curva de calibración entre una cantera y otra.

Este sistema de sensado presenta un alto potencial para ser usado para medir el $\mathrm{CH}$ en $\mathrm{ADC}$ en el proceso de fabricación de concreto.

\section{Agradecimientos}

Este artículo ha sido financiado por Concytec y Sencico, en el proyecto «Tecnologías de facilitación basadas en técnicas de microondas para la medición en tiempo real del contenido de humedad en materiales de construcción» - Contrato 108-2017.

\section{Referencias}

[1] J. B. Hasted and M. A. Shah, "Microwave absorption by water in building materials," British Journal of Applied Physics, vol. 15, no. 7, pp. 825-836, jul 1964. [Online]. Available: https://doi. org $/ 10.1088 \% 2$ F0508-3443\%2F15\%2F7\%2F307

[2] A. Cataldo, E. De Benedetto, and G. Cannazza, "Hydration monitoring and moisture control of cement-based samples through embedded wire-like sensing elements," IEEE Sensors Journal, vol. 15, no. 2, pp. 1208-1215, 2015. [Online]. Available: https: //doi.org/10.1109/JSEN.2014.2360712

[3] F. P. Balobey, "Optimalisation of microwave method for moisture content measurement in asbestos-cement sheets," Russian: Oborudovanye Prom. Story. Meter, Instrum. for Build. Mater, vol. 1, pp. 44-50, 1964. 
[4] A. W. Kraszewski, "Microwave aquametryneeds and perspectives," IEEE Transactions on Microwave Theory and Techniques, vol. 39, no. 5, pp. 828-835, 1991. [Online]. Available: https://doi.org/10.1109/22.79110

[5] A. Kraszewski, "Microwave aquametry-a bibliography," Journal of Microwave Power, vol. 15, no. 4, pp. 298-310, 1980. [Online]. Available: https: //doi.org/10.1080/16070658.1980.11689215

[6] V. Komarov, Handbook of Dielectric and Thermal Properties of Materials at Microwave Frequencies. Artech House, 2012. [Online]. Available: https://bit.ly/2WQE2Tv

[7] A. Cownie and L. S. Palmer, "The effect of moisture on the electrical properties of soil," Proceedings of the Physical Society. Section $B$, vol. 65, no. 4, pp. 295-301, apr 1952. [Online]. Available: https://doi.org/10.1088\% 2F0370-1301\%2F65\%2F4\%2F308

[8] J. J. Windle and T. M. Shaw, "Dielectric properties of wool-water systems. ii. 26000 megacycles," The Journal of Chemical Physics, vol. 25, no. 3, pp. 435-439, 1956. [Online]. Available: https://doi.org/10.1063/1.1742941

[9] A. Nakanishi, T. Hori, and J. Fujiwara, "An evaluation of "moister"as a measuring apparatus of the moisture content of paper," Japanese: Res. Inst. Techn. Bull, vol. 1, pp. 9-20, 1955.

[10] A. Yasukawa, "Measurement and automatic control of moisture content in rayon pulp: Soc. of instrum," Technol. Japan J, vol. 6, no. 8, pp. 386391, 1956.

[11] R. Rodríguez Arisméndiz, "Estudio de la espectroscopía dieléctrica para la medición del contenido de humedad en productos alimenticios," Ph.D. dissertation, 2017. [Online]. Available: https://hdl.handle.net/11042/3487

[12] M. Berliner and S. A. Polishchuk, "Instrument for moisture content measurement in slurries," Russian: Cement, pp. 14-15, 1978.

[13] D. K. Cheng, Fundamentos de electromagnetismo para ingeniería. Pearson Educación, 1997. [Online]. Available: https://bit.ly/3cvOXIP

[14] A. R. Dean and P. Bridle, "Test on the use of a microwave moisture meter," Timber Trade J., vol. 27, pp. 50-52, 1969.

[15] D. F. Tirado, P. M. Montero, and D. Acevedo, "Estudio comparativo de métodos empleados para la determinación de humedad de varias matrices alimentarias," Información tecnológica, vol. 26, pp. 03-10, 00 2015. [Online]. Available: http: //dx.doi.org/10.4067/S0718-07642015000200002

[16] G. R. Oetzel, F. P. Villalba, W. J. Goodger, and K. V. Nordlund, "A comparison of on-farm methods for estimating the dry matter content of feed ingredients1," Journal of Dairy Science, vol. 76, no. 1, pp. 293-299, 1993. [Online]. Available: https: //doi.org/10.3168/jds.S0022-0302(93)77349-X

[17] L. Jílková, T. Hlinčík, and K. Ciahotný, "Determination of water content in pyrolytic tars using coulometric karl-fischer titration," Journal of Advanced Engineering, vol. 57, no. 1, pp. 8-13, 2017. [Online]. Available: http://dx.doi.org/10.14311/AP.2017.57.0008

[18] A. M. Helmenstine, "What is distillation? chemistry definition," ThoughtCo, 2019. [Online]. Available: https://bit.ly/2WLgTli

[19] L. M. L. Nollet, Handbook of Food Analysis: Physical characterization and nutrient analysis, ser. Food Science and Technology - Marcel Dekker, Inc. CRC PressI Llc, 2004. [Online]. Available: https://bit.ly/3fMQrAI

[20] G. S. Campbell and C. S. Campbell, "Water content and potential, measurement," in Reference Module in Earth Systems and Environmental Sciences. Elsevier, 2013. [Online]. Available: https: //doi.org/10.1016/B978-0-12-409548-9.05333-1

[21] M. R. Goyal, Management of Drip/Trickle or Micro Irrigation. Apple Academic Press, 2012. [Online]. Available: https://bit.ly/2yJGaVl

[22] T. Reyna, J. Linares, M. Lábaque, and S. Reyna, "Métodos para medir el contenido de humedad vs. el tiempo. estudios de infiltración. evaluación de resultados de campo," in XXVII Congreso Latinoamericano de Hidráulica, Lima Perú, 09 2016. [Online]. Available: https://bit.ly/3dIYexC

[23] S. Jiménez, L. Scarioni, and H. Kelim, "Nota técnica: Sensores de humedad de tipo capacitivo y resistivo, fabricados con $\mathrm{NaCl}, \mathrm{KBr}$ y $\mathrm{KCl}$," INGENIERIA UC, vol. 20, no. 1, pp. 83-86, 04 2013. [Online]. Available: https://bit.ly/2Wrj2Uv

[24] S. Okamura, "Microwave technology for moisture measurement," Subsurface Sensing Technologies and Applications, vol. 1, no. 2, pp. 205-227, 2000. [Online]. Available: https://bit.ly/2WWETC6

[25] E. Pinos-Vélez, S. Encalada, E. Gamboa, V. Robles-Bykbaev, W. Ipanque, and C. L. Chacón, "Development of a support system for the presumptive diagnosis of glaucoma through the processing of biomedical images of the human eye fundus in Ecuador," in Advances in Human Factors and Ergonomics in 
Healthcare and Medical Devices, V. Duffy and N. Lightner, Eds. Cham: Springer International Publishing, 2018, pp. 100-109. [Online]. Available: https://doi.org/10.1007/978-3-319-60483-1_11

[26] J. Soto, E. Paiva, W. Ipanaqué, J. Reyes, D. Espinoza, and D. Mendoza, "Cocoa bean quality assessment by using hyperspectral index for determining the state of fermentation with a nondestructive analysis," in 2017 CHILEAN Conference on Electrical, Electronics Engineering, Information and Communication Technologies (CHILECON), 2017, pp. 1-5. [Online]. Available: https: //doi.org/10.1109/CHILECON.2017.8229718

[27] J. M. Ruiz Reyes, J. Soto Bohorquez, and W. Ipanaque, "Evaluation of spectral relation indexes of the peruvian's cocoa beans during fermentation process," IEEE Latin America Transactions, vol. 14, no. 6, pp. 2862-2867, 2016. [Online]. Available: https://doi.org/10.1109/TLA.2016.7555266

[28] J. Soto, J. Ruiz, W. Ipanaqué, and C. Chinguel, "New hyperspectral index for determining the state of fermentation in the non-destructive analysis for organic cocoa violet," in 2016 IEEE International Conference on Automatica (ICAACCA ), 2016, pp. 1-6. [Online]. Available: https: //doi.org/10.1109/ICA-ACCA.2016.7778387

[29] J. Soto, G. Granda, F. Prieto, W. Ipanaque, and J. Machacuay, "Cocoa bean quality assessment by using hyperspectral images and fuzzy logic techniques," in Twelfth International Conference on Quality Control by Artificial Vision 2015, F. Meriaudeau and O. Aubreton, Eds., vol. 9534, International Society for Optics and Photonics. SPIE, 2015, pp. 152-158. [Online]. Available: https://doi.org/10.1117/12.2182598

[30] A. W. Kraszewski and S. O. Nelson, "Microwave resonator technique for moisture content and mass determination in single soybean seeds," IEEE Transactions on Instrumentation and Measurement, vol. 43, no. 3, pp. 487-489, 1994. [Online]. Available: https://doi.org/10.1109/19.293475

[31] P. G. Bartley, S. O. Nelson, R. W. McClendon, and S. Trabelsi, "Determining moisture content of wheat with an artificial neural network from microwave transmission measurements," IEEE Transactions on Instrumentation and Measurement, vol. 47, no. 1, pp. 123-126, 1998. [Online]. Available: https://doi.org/10.1109/19.728803

[32] K.-B. Kim, J.-H. Kim, S. S. Lee, and S. H. Noh, "Measurement of grain moisture content using microwave attenuation at $10.5 \mathrm{GHz}$ and moisture density," IEEE Transactions on Instrumentation and Measurement, vol. 51, no. 1, pp. $72-77,2002$. [Online]. Available: https://doi.org/10.1109/19.989904

[33] M. Ben Slima, R. Z. Morawski, A. W. Kraszewski, A. Barwicz, and S. O. Nelson, "Calibration of a microwave system for measuring grain moisture content," IEEE Transactions on Instrumentation and Measurement, vol. 48, no. 3, pp. 778-783, 1999. [Online]. Available: https://doi.org/10.1109/19.772221

[34] S. Trabelsi and S. O. Nelson, "Free-space measurement of dielectric properties of cereal grain and oilseed at microwave frequencies," Measurement Science and Technology, vol. 14, no. 5, pp. 589600, mar 2003. [Online]. Available: https://doi. org/10.1088\%2F0957-0233\%2F14\%2F5\%2F308

[35] C. V. K. Kandala, "Moisture determination in single peanut pods by complex rf impedance measurement," IEEE Transactions on Instrumentation and Measurement, vol. 53, no. 6 , pp. 1493-1496, 2004. [Online]. Available: https://doi.org/10.1109/TIM.2004.834058

[36] Z. Abbas, You Kok Yeow, A. H. Shaari, K. Khalid, J. Hassan, and E. Saion, "Complex permittivity and moisture measurements of oil palm fruits using an open-ended coaxial sensor," IEEE Sensors Journal, vol. 5, no. 6 , pp. $1281-1287,2005$. [Online]. Available: https://doi.org/10.1109/JSEN.2005.859249

[37] S. Trabelsi and S. O. Nelson, "Influence of nonequilibrated water on microwave dielectric properties of wheat and related errors in moisture sensing," IEEE Transactions on Instrumentation and Measurement, vol. 56, no. 1, pp. 194-198, 2007. [Online]. Available: https://doi.org/10.1109/TIM.2006.887314

[38] K. Tsukada and T. Kiwa, "Magnetic measurement of moisture content of grain," IEEE Transactions on Magnetics, vol. 43, no. 6, pp. 2683-2685, 2007. [Online]. Available: https://doi.org/10.1109/TMAG.2007.892853

[39] C. V. K. Kandala and S. O. Nelson, "Rf impedance method for estimating moisture content in small samples of in-shell peanuts," IEEE Transactions on Instrumentation and Measurement, vol. 56, no. 3, pp. 938-943, 2007. [Online]. Available: https://doi.org/10.1109/TIM.2007.894796

[40] C. V. Kandala and N. Puppala, "Parallel-plate capacitance sensor for nondestructive measurement of moisture content of different types of wheat," 
in 2012 IEEE Sensors Applications Symposium Proceedings, 2012, pp. 1-5. [Online]. Available: https://doi.org/10.1109/SAS.2012.6166325

[41] S. T. Wahyuni Siregar, W. Handayani, and A. H. Saputro, "Bananas moisture content prediction system using visual-nir imaging," in 2017 5th International Conference on Instrumentation, Control, and Automation (ICA), 2017, pp. 89-92. [Online]. Available: https://doi.org/10.1109/ICA.2017.8068419 